\title{
Synthesis of Hydroxyapatite/Collagen (HA/COL) Composite Powder Using a Novel Precipitation Technique
}

\author{
A.B.H. YoruC ${ }^{a, *}$, A.K. AydinOGLU ${ }^{b}$ \\ ${ }^{a}$ Yildiz Technical University, Department of Metallurgical and Materials Engineering, \\ Davutpaşa Campus, 34210, Esenler, Istanbul, Turkey \\ ${ }^{b}$ Yildiz Technical University, Department of Bioengineering, Davutpaşa Campus, 34210, Esenler, Istanbul, Turkey
}

\begin{abstract}
A new biomimetic precipitation technique was developed to synthesize hydroxyapatite/collagen nanocomposites. The componential and morphological properties of nano-composites were investigated. It was revealed that the inorganic phase in the nano-composite was carbonate-substituted hydroxyapatite with low crystallinity. Indeed both characteristic peaks for $\mathrm{HA}$ and $\mathrm{Col}$ molecule and shifts of the characteristic peaks have indicated on HA-Col bonding. Morphology studies showed that hydroxyapatite particles with the average size of $50 \mathrm{~nm}$ were distributed homogeneously in the polymer matrix. According to the TEM micrographs, inorganic particles were needle-like and had regular crystallographic orientation. These results suggest that the hydroxyapatite/collagen nano-composites are promising biomaterials for bone tissue engineering.
\end{abstract}

DOI: $10.12693 /$ APhysPolA.127.1264

PACS: 7.85.J

\section{Introduction}

Bone repair or regeneration is a key problem in orthopedic surgery. In the cases of severe trauma or disease, surgical intervention is required. Bone grafts and bone graft substitutes are used in the surgical repair and reconstruction of critical defects. Autografts have achieved various degrees of success in treating bone defects. However, the autograft is limited by the donor site morbidity, prolonged rehabilitation, increased risk of deep infection and restricted availability. Moreover, allografts might cause potential risks of transmitted diseases such as HIV or contamination $[1,2]$. Bone tissue engineering aims to develop bone graft replacements that can repair bone defects without a need for autografts [2]. Bone is a structural biological composite made of a mineral part, consisting of hydroxyapatite (HA) as a main component of mineral matrix, and an organic part, mainly consisting of collagen molecules.

Hydroxyapatite and collagen represent the basic building blocks of bone that are arranged at various lengthscales to form a complex hierarchical structure $[3,4]$. Hydroxyapatite $\left[\mathrm{Ca}_{10}\left(\mathrm{PO}_{4}\right)_{6}(\mathrm{OH})_{2}\right]$ exhibits excellent biocompatibility, bioactivity, strong plasticity, outstanding mechanical properties and osteoconductivity in vivo. Collagen (Col) is widely used for making tissue scaffolds, because of its predominance in the extracellular matrix $(\mathrm{ECM})$, non-immunogenicity and available methods of isolation from a variety of sources. In addition, collagen is resorbable with excellent biocompatibility and ability to promote tissue regeneration $[5,6]$. When combined with $\mathrm{HA}$, the hydrophilic nature of Col could improve the wettability and permeability of materials, re-

* corresponding author; e-mail: afife.hazar@gmail.com sulting in culture medium penetration that is favorable for cell adhesion [6]. The collagen molecules are parallel to each other and staggered along the length by $67 \mathrm{~nm}$, which results in formation of $40 \mathrm{~nm}$ long hole-zones and $27 \mathrm{~nm}$ long overlap-zones. The mineral component of bone (HA) is oriented in such way, that its $c$-axis is parallel to the length of collagen. The HA crystal platelets nucleate in hole-zones, and grow in length along collagen long axis, and in width along channels. The crystals continue to grow, out-growing the width of hole-zone and into the overlap-zone, squeezing in between the collagen molecules. In addition to intra fibrillar mineral the surface of fibril is coated by extra fibrillar mineral [7]

Currently, there are some techniques concerning preparation of $\mathrm{HA} / \mathrm{Col}$ composite materials, including coprecipitation, alternate soaking and mechanical mixing. Among these methods, there is a common shortcoming, that inorganic particles cannot be distributed homogeneously in the organic matrices at nanolevel, which leads to poor mechanical properties and limits their applications [1]. In this study, collagen molecule was prefibrillated and then $\mathrm{HA} / \mathrm{Col}$ nano-composites were synthesized by new biomimetic co-precipitation technique with the humidity conditions $(\mathrm{pH}$ value $7.4-8.0$ and at $\left.37^{\circ} \mathrm{C}\right)$.

\section{Materials and methods}

Soluble type-I collagen from bovine skin was purchased from Leather and Footwear Research Institute, Collagen Department (Ukraine). Calcium nitrate tetra hydrate $\left[\mathrm{Ca}\left(\mathrm{NO}_{3}\right)_{2} \cdot 4 \mathrm{H}_{2} \mathrm{O}\right]$, diammonium hydrogen phosphate $\left[\left(\mathrm{NH}_{4}\right)_{2} \mathrm{HPO}_{4}\right]$ were purchased from SigmaAldrich. All the other reagents were purchased from Merck. All the reagents used in this work were of analytical grade (AR) and used without any further purification. Ultrapure water and simulated body fluids $[8,9]$ were used throughout the experiment. 
In this study to mimic the natural formation of bone matrix, collagen was fibrillated by following methods: In the first method, collagen-phosphate buffer solution (4:1) was prepared on ice bath and then $\mathrm{pH}$ was adjusted to 9 with $0.1 \mathrm{M} \mathrm{NaOH}$ solution and incubated at $37{ }^{\circ} \mathrm{C}$. Micro-fibrillation was determined at $400 \mathrm{~nm}$ by UV-Vis spectrophotometer (PGENERAL, T80+) and results showed that fibrillation was completed in $3.5 \mathrm{~h}$. In the second method, collagen was dissolved in the ultrapure water and $\mathrm{pH}$ was adjusted to 3.5 . After that $1.3 \mathrm{M}$ $\mathrm{NaCl}$ solution was added to this solution under vigorous stirring, micro-fibrillation was investigated at $400 \mathrm{~nm}$ to determine the optimum $\mathrm{NaCl}$ volume. Resulted solution was centrifuged at $1300 \mathrm{rpm}$ and $\mathrm{pH}$ was adjusted to 9.0 by $0.01 \mathrm{M} \mathrm{NH}_{4} \mathrm{OH}$ solution. Fibrillated collagen has been called FCol-1 and FCol-2, respectively.

Hydroxyapatite/Collagen (HA/Col) composite powder was synthesized by two different co-precipitation method. In the first method, after $1.1 \mathrm{M}$ of $\mathrm{Ca}\left(\mathrm{NO}_{3}\right)_{2} \cdot 4 \mathrm{H}_{2} \mathrm{O}$ solution was added to $50 \mathrm{~mL}$ collagen (15 wt.\%) solution and stirred for $24 \mathrm{~h}, 0.7 \mathrm{M}$ of $\left(\mathrm{NH}_{4}\right)_{2} \mathrm{HPO}_{4}$ solution was added to this mixture. In the second method, $50 \mathrm{ml}$ of collagen $(15 \mathrm{wt} . \%)$ solution and $0.7 \mathrm{M}$ of $\left(\mathrm{NH}_{4}\right)_{2} \mathrm{HPO}_{4}$ solution were stirred on the ice bath. Resulted mixture was degassed and $\mathrm{pH}$ was adjusted to 9 by $1 \mathrm{M} \mathrm{NaOH}$ solution. $1.1 \mathrm{M}$ of $\mathrm{Ca}\left(\mathrm{NO}_{3}\right)_{2} \cdot 4 \mathrm{H}_{2} \mathrm{O}$ solution was added this mixture. Resulted mixtures were aged for $24 \mathrm{~h}$ at $37{ }^{\circ} \mathrm{C}$, filtrated and dried under vacuum at $37{ }^{\circ} \mathrm{C}$, respectively. In all steps temperature was kept at $37{ }^{\circ} \mathrm{C}$ and $\mathrm{pH}$ was adjusted to 9 by $1 \mathrm{M}$ $\mathrm{NaOH}$ solution. Simulated body fluids were used as reaction media as well. Based on the synthesis method, resulted composite powders were called $\mathrm{HA} / \mathrm{Col}-1$ and HA/Col-2, respectively. The crystalline phase and components of obtained products were identified using wide angle X-ray diffraction analysis (Shimadzu, XRD-2000) and Fourier Transform Infrared Spectrometer (FT-IR-Perkin-Elmer Spectrum-100). Morphology of inorganic/organic composite was observed using Environmental Scanning Electron Microscopy-SEM (JEOL-JSM-6335F) and High Resolution Transmission Electron Microscope-TEM (JEOL-2100 HRTEM).

\section{Results and discussion}

As mentioned before collagen was fibrillated by two different methods. Absorbance of the solutions was measured as a function of time and as function of added $\mathrm{NaCl}$ amount. Absorption values of FCol-1 and FCol-2 are shown in Fig. 1. Second method was chosen to fibrillation of collagen due to the higher absorbance and yield, low cost and easier application.

FT-IR spectroscopy is a good structural characterization method, for HA/Col composites. Figure 2 shows the FT-IR spectra of HA/Col-1 and HA/Col-2. The bands at $1092,1035,961,603$ and $566 \mathrm{~cm}^{-1}$ corresponded to different vibration modes of phosphate group in HA, while the bands at 3570 and $632 \mathrm{~cm}^{-1}$ represent the stretching and bending vibration of hydroxyl group. Bands assigned to carbonate group at 1482, 1452, 1424 and $874 \mathrm{~cm}^{-1}$ were also observed. This is in sgreement with the fact that HA crystals prepared using the precipitation method, contained carbonate ions, similar to the natural bone tissue. As shown in Fig. 1, the typical amide peaks of the type-I Col appear at $1639 \mathrm{~cm}^{-1}$ (amide I), 1551 $\mathrm{cm}^{-1}$ (amide II), $1238 \mathrm{~cm}^{-1}$ (amide III). The peak at $3080 \mathrm{~cm}^{-1}$ belongs to the stretching vibration of amide hydrogen bonding (NH) [10, 11-13]. FT-IR spectrums have proved that formation of $\mathrm{HA} / \mathrm{Col}$ composite structures has ocurred.
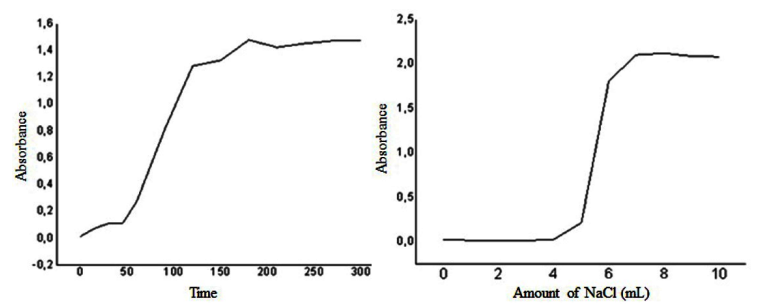

Fig. 1. Absorbance values of FCol-1 and FCol-2 solutions.

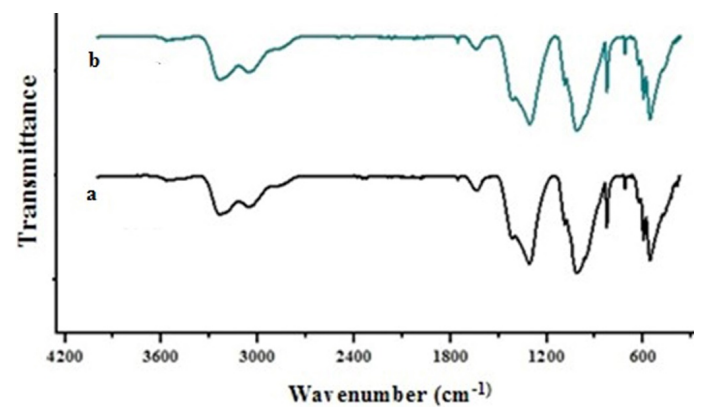

Fig. 2. FT-IR spectrums of (a) HA/Col-1 and (b) $\mathrm{HA} / \mathrm{Col}-2$ composite powders.

The crystallographic structure of the HA/Col composites prepared in this study is shown in Fig. 3. It can be seen that poorly crystalline $\mathrm{HA}$ can be readily discerned in all samples. No peaks associated with the reactants are present, which shows that complete reaction of the calcium phosphate precursors takes place, under the given conditions, for all studied compositions. Three intense peaks of crystal phases at $25.9^{\circ}, 32^{\circ}$ and $39.7^{\circ}$ $(2 \theta)$, which are assigned to $(002),(211)$ and (310) reflections of crystalline HA, are clearly seen. This crystallographic structure of the samples is similar to natural bone mineral (biological apatite). In addition, another intense peak of $\mathrm{HA} / \mathrm{Col}-2$ pattern at $34^{\circ}(2 \theta)$ is sharper than that of $\mathrm{HA} / \mathrm{Col}-1$ and pure hydroxyapatite, which is associated with the orientation of hydroxyapatite crystals, which is the effect of the collagen fibril orientation. XRD pattern of HA/Col-1 and HA/Col-2 composite was also confirmed by FT-IR analysis (Fig. 1) [1, 10, 12, 13].

Figure 4 shows SEM micrographs of $\mathrm{HA} / \mathrm{Col}-1$ and HA/Col-2 nano-composites. Through the observation of higher magnification SEM micrographs (Fig. 4b and 4c), the morphological features have revealed that inorganic crystals of HA had a high-affinity with the organic matrices in all samples. The inorganic particles 
are distributed within Col matrix homogenously. Thus, this close bonding between inorganic particles and organic matrix may enhance the mechanical properties of the composites $[11,13]$. However, it is hard to get this kind of decentralization effect by conventional mechanical mixing or co-precipitation. Also formation of collagen fibrillation has been clearly seen from the SEM micrographs. However there has been no significant difference between HA/Col-1 and HA/Col-2 structure, which shows that precipitation temperature has not affected the morphology of final product.

Highly magnified TEM images of samples are shown in Fig. 5. They indicate that composites have nanoscale sub-crystallites in organic matrices, but do not have a uniform crystallographic orientation. However $\mathrm{HA} / \mathrm{Col}-1$ composite is more crystallographically orientted than the HA/Col-2 composite. The inorganic particles with average size of $50 \mathrm{~nm}$ are distributed more homogenously within $\mathrm{HA} / \mathrm{Col}-1$ matrix, compared with HA/Col-2 matrix.

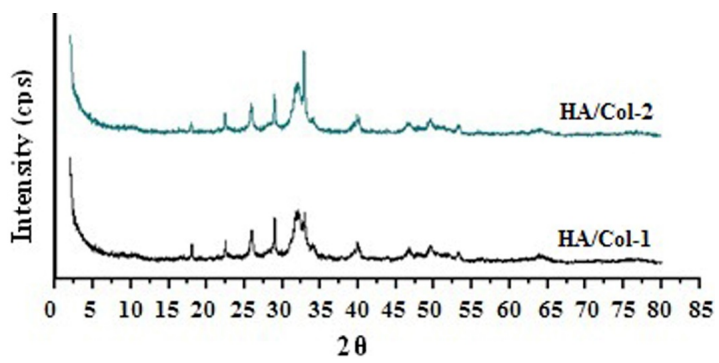

Fig. 3. XRD patterns of $\mathrm{HA} / \mathrm{Col}-1$ and $\mathrm{HA} / \mathrm{Col}-2$ composites.

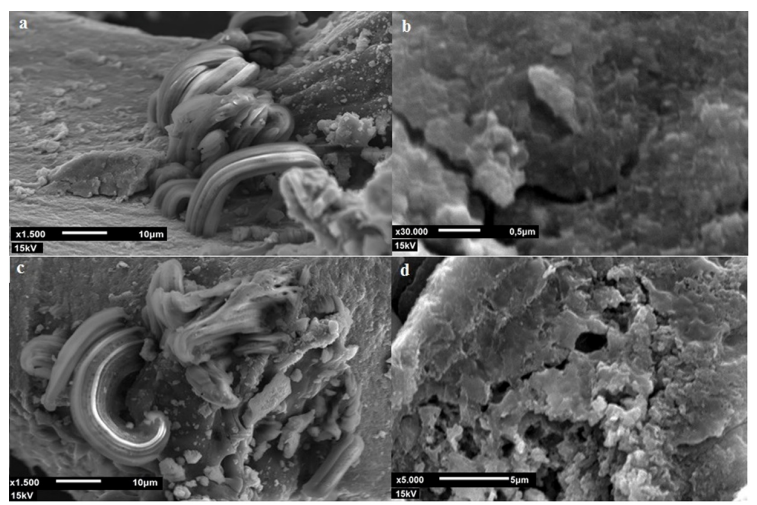

Fig. 4. SEM micrographs of (a, b) HA/Col-1 and (c, d) $\mathrm{HA} / \mathrm{Col}-2$.

\section{Conclusions}

Hydroxyapatite/collagen nano-composite powder was obtained via a new biomimetic precipitation method. The yield of the $\mathrm{HAp} / \mathrm{Col}$ composite is closely related to the $\mathrm{pH}$ value during the preparation. Higher $\mathrm{pH}$ favors the formation of composite. In addition, the $\mathrm{pH}$ value is detrimental for the hydrogen bonding between collagen fibrils/fibers, as well as the organic-inorganic phases. The inorganic component in the composite was identified as mono-phase, low crystalline HA, containing carbonate ions. FT-IR results show both characteristic peaks for $\mathrm{HA}$ and Col molecule and the shift of the characteristic peaks indicates HA-Col bonding. XRD results show that HA has a selected orientation which is affected by the collagen molecules. In the XRD pattern of HA/Col composite, $34^{\circ}$ peak is sharper than that on the pattern of pure HA. In addition, the organic matrix has been fibrillated and the HA crystals have been oriented under the effect of collagen matrix. The crystalline structure of HA can be readily discerned, for all samples, as is illustrated by the XRD patterns. The morphology of the HA/Col composites exhibits a needle-like structure. The inorganic particles with average size $50 \mathrm{~nm}$ were distributed within organic matrix homogenously. From these results it can be concluded that $\mathrm{HA} / \mathrm{Col}$ composites can be synthesized, in vitro, under conditions native to human physiology. The desirable conditions for $\mathrm{HA} / \mathrm{Col}$ synthesis, in vitro, are similar, with $\mathrm{pH}$ of ( 7 to 8$)$ and a temperature of $37^{\circ} \mathrm{C}$. Biocompatibility of $\mathrm{HA} / \mathrm{Col}$ composite as a graft material for the repair bone defect regeneration will be investigated. Based on the above research, we make a conclusion that the $\mathrm{HA} / \mathrm{Col}$ nano-composites are promising biomaterials for bone tissue engineering.

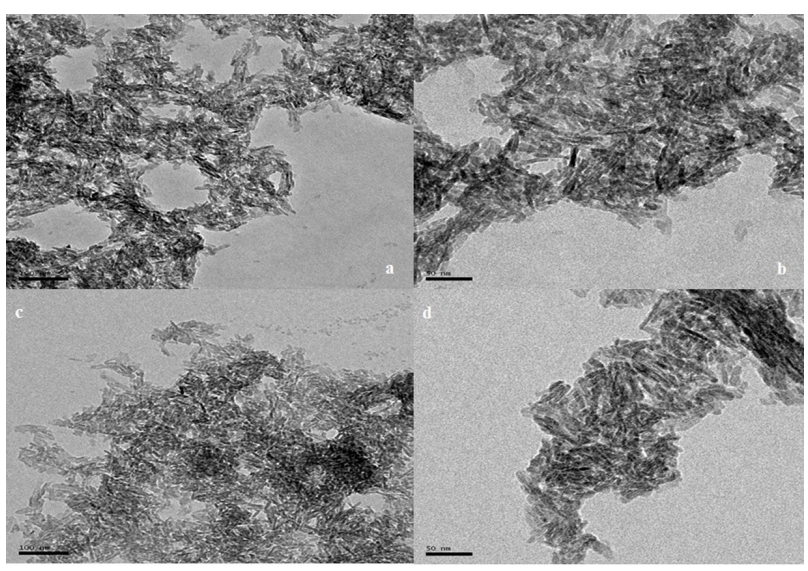

Fig. 5. TEM images of (a) HA/Col-1 composite at $100 \mathrm{~nm}$ scale, (b) HA/Col-1 composite at $50 \mathrm{~nm}$ scale, (c) $\mathrm{HA} / \mathrm{Col}-2$ composite at $100 \mathrm{~nm}$ scale and (d) $\mathrm{HA} / \mathrm{Col}-2$ composite at $50 \mathrm{~nm}$ scale.

\section{Acknowledgments}

This study was supported by the Scientific and Technological Research Council of Turkey within the scope of the project nr. $110 \mathrm{M} 182$.

\section{References}

[1] L. Chen, J. Hu, J. Ran, X. Shen, H. Tong, International Journal of Biological Macromolecules 65, 1 (2014).

[2] C.M. Murphy, A. Schindeler, J.P. Gleeson, N.Y.C. Yu, L.C. Cantril, K. Mikulec, L. Peacock, F.J. O'Brien, D.G. Little, Acta Biomaterialia 10, 2250 (2014).

[3] F. Libonati, A.K. Nair, L. Vergani, M.J. Buehler, Mechanics Research Communications 58, 17 (2014). 
[4] R.J. Kane, R.K. Roeder, Journal of The Mechanical Behavior of Biomedical Materials 7, 41 (2012).

[5] C.C. Yu, J.J. Chang, Y.H. Lee, Y.C. Lin, M.H. Wu, M.C. Yang, C.T. Chien, Materials Letters 93, 133 (2013).

[6] L. Jia, Z. Duan, D. Fan, Y. Mi, J. Hui, L. Chang, Materials Science and Engineering C 33, 727 (2013).

[7] D.R. Katti, S.M. Pradhan, K.S. Katti, Journal of Biomechanics 43, 1723 (2010).

[8] A.C. Tas, Journal of the European Ceramic Society 20, 2389 (2000).
[9] A.C. Tas, F. Korkusuz, M. Timucin, N. Akkas, J. Mater. Sci.: Mater. Med. 8, 91 (1997).

[10] A. Ficai, E. Andronescu, G. Voicu, C. Ghitulica, D. Ficai, Materials Characterization 61, 402 (2010).

[11] A. Ficai, E. Andronescu, V. Trandafir, C. Ghitulica, G. Voicu, Materials Letters 64, 541 (2010).

[12] A. Sionkowska, J. Kozłowska, International Journal of Biological Macromolecules 47, 483 (2010).

[13] L. Zhang, P. Tang, M. Xu, W. Zhang, W. Chai, Y. Wang, Acta Biomaterialia 6, 2189 (2010). 